Communications in Physics, Vol. 26, No. 1 (2016), pp. 11-18

DOI:10.15625/0868-3166/26/1/7790

\title{
RIPPLON MODES OF TWO SEGREGATED BOSE-EINSTEIN CONDENSATES IN CONFINED GEOMETRY
}

\author{
TRAN HUU PHAT \\ Vietnam Atomic Energy Commission, 59 Ly Thuong Kiet, Hanoi, Vietnam \\ HOANG VAN QUYET ${ }^{\dagger}$ \\ Department of Physics, Hanoi Pedagogical University No. 2, Hanoi, Vietnam \\ ${ }^{\dagger} E$-mail: hoangvanquyetsp2@gmail.com
}

Received 20 February 2016

Accepted for publication 12 April 2016

\begin{abstract}
The ripplon modes of two segregated Bose-Einstein condensates (BECs) confined by one and two hard walls are respectively studied by means of the hydrodynamic approach within the Gross-Pitaevskii (GP) theory. For the system at rest we find that due to the spatial restriction the dispersion relations are of the form $\omega \sim k^{2}$ in low momentum limit for both cases, while for the system in motion parallel to the interface the dispersion relations for both cases are $\omega \sim k$ at low momentum limit and, furthermore, the system becomes unstable.
\end{abstract}

Keywords: Bose-Einstein condensates, hydrodynamic approach, ripplon modes, Kelvin-Helmholtz instability, Bernoulli equation.

Classification numbers: 03.75.Fi, 05.30.Jp, 67.60.- g.

\section{INTRODUCTION}

The phase separation in binary mixtures of Bose-Einstein condensates was theoretically predicted [1,2] and observed later in experiments [3-8]. Since then many works have been devoted to explore the statics, the dynamics and the ripplon modes of two segregated BECs [9-25]. However, most of them concerned with the systems in infinite space, while all experimental realizations have been carried out in restricted regions of space with spatial structure being more and more complicated. In this respect, the present paper deals with the problem: how ripplon modes are influenced by the spatial restriction when a system of two segregated BECs is confined by one or two hard walls and the method we use to tackle the problem is the hydrodynamic approach. To 
begin with, let us start from the GP Lagrangian

$$
\begin{gathered}
£=\int d \vec{r}\left(P_{1}+P_{2}-g_{12}\left|\psi_{1}\right|^{2}\left|\psi_{2}\right|^{2}\right), \\
P_{j}=i \hbar \psi_{j}^{*} \frac{\partial \psi_{j}}{\partial t}+\frac{\hbar^{2}}{2 m_{j}}\left|\nabla \psi_{j}\right|^{2}-\frac{g_{j j}}{2}\left|\psi_{j}\right|^{4},
\end{gathered}
$$

with $m_{j}$ being the atomic mass of the $\mathrm{j}$ component. The intra-s and inter-species interaction coupling constants are defined as

$$
g_{j k}=2 \pi \hbar^{2} a_{j k}\left(m_{j}^{-1}+m_{k}^{-1}\right),
$$

where $a_{j k}$ is the scattering length between the atoms in components $j$ and $k$. In the following we assume that two BECs are separated $g_{12}^{2}>g_{11} g_{22}$.

From the Lagrangian (1) the GP equations are deduced straightforwardly

$$
\begin{aligned}
& i \hbar \frac{\partial \psi_{1}}{\partial t}=\left(-\frac{\hbar^{2}}{2 m_{1}} \nabla^{2}+g_{11}\left|\psi_{1}\right|^{2}+g_{12}\left|\psi_{2}\right|^{2}\right) \psi_{1}, \\
& i \hbar \frac{\partial \psi_{2}}{\partial t}=\left(-\frac{\hbar^{2}}{2 m_{2}} \nabla^{2}+g_{22}\left|\psi_{2}\right|^{2}+g_{12}\left|\psi_{1}\right|^{2}\right) \psi_{2} .
\end{aligned}
$$

This paper is organized as follows. The main body of the work is presented in Sec. II where the Bernoulli equation is established and the ripplon modes are derived. The conclusion and discussion are given in Sec. III.

\section{RIPPLON MODES}

First of all let us establish the Bernoulli equation for the system of two BECs separated by the interface which is represented by the equation $z=z_{0}+\eta(\sigma), \sigma=\left(k_{x} x+k_{y} y-\omega t\right)$ and located at $z=z_{0}$. Assume that the component 1 (component 2) resides in the region $z>z_{0}\left(z<z_{0}\right)$. Then the Lagrangian (1a) is approximated by

$$
£=2 \pi \int d x d y\left(\int_{-\infty}^{\eta+z_{0}} d z P_{2}+\int_{\eta+z_{0}}^{+\infty} d z P_{1}\right)-\alpha S
$$

where $\alpha$ is the interface tension and the interface area $\mathrm{S}$ is given by

$$
S=\int d x d y\left[1+\left(\frac{\partial \eta}{\partial x}\right)^{2}+\left(\frac{\partial \eta}{\partial y}\right)^{2 \frac{1}{2}} \approx \int d x d y\left[1+\frac{1}{2}\left(\frac{\partial \eta}{\partial x}\right)^{2}+\frac{1}{2}\left(\frac{\partial \eta}{\partial y}\right)^{2}\right]\right.
$$

for interface with negligible thickness. From (3) and (4) we arrive at the Bernoulli equation

$$
P_{1}\left(x, y, z=z_{0}+\eta, t\right)-P_{2}\left(x, y, z=z_{0}+\eta, t\right)=\alpha\left(\frac{\partial^{2}}{\partial x^{2}}+\frac{\partial^{2}}{\partial y^{2}}\right),
$$

which corresponds to the classic Bernoulli equation in hydrodynamics [26,27]. Next, we proceed to the GP equations in linear approximation as made in Ref. [17] writing

$$
\psi_{j}(\mathrm{x}, \mathrm{y}, \mathrm{z}, \mathrm{t},)=\sqrt{n_{j}(\mathrm{x}, \mathrm{y}, \mathrm{z}, \mathrm{t})} e^{i \phi_{j}(\mathrm{x}, \mathrm{y}, \mathrm{z}, \mathrm{t})},
$$


in which the particle number density and phase, $n_{j}(x, y, z, t)$ and $\phi_{j}(x, y, z, t)$, are decomposed as

$$
\begin{gathered}
n_{j}(x, y, z, t)=n_{j 0}+\delta n_{j}(x, y, z, t), \\
\phi_{j}(x, y, z, t)=-\frac{g_{j j} n_{j 0}}{\hbar} t+\delta \phi_{j}(x, y, z, t) .
\end{gathered}
$$

Inserting (6) and (7) into (2) and taking only the first order of $\delta n_{j}, \delta \phi_{j}$ we are led to the approximate equations

$$
\begin{gathered}
\nabla \overrightarrow{v_{j}}=0, \\
\hbar \frac{\partial}{\partial \mathrm{t}}\left(\delta \phi_{\mathrm{j}}\right)+\mathrm{g}_{j j} \delta \mathrm{n}_{\mathrm{j}}=0,
\end{gathered}
$$

assuming that the relative density changes following fluid particles are small compared to the velocity gradient [28]. The velocity $\vec{v}_{j}$ in (8a) is defined as

$$
\overrightarrow{v_{j}}=\frac{\hbar}{m_{j}} \vec{\nabla} \delta \phi_{j}
$$

Eq. (8a) tells that in the approximation under consideration the condensates are incompressible fluid.

Based on the approximate equations (8) and (9) the pressure given in (1b) takes the form

$$
P_{j}=\frac{1}{2} g_{j j} n_{j 0}^{2}+g_{j j} n_{j 0} \delta n_{j}
$$

To solve (8a) and (9) we adopt the ansatz

$$
\delta \phi_{j}(x)=\varphi_{j}(z) \chi_{j}(\sigma)
$$

from which we get the equations

$$
\begin{gathered}
\left(\frac{d^{2}}{d z^{2}}-k^{2}\right) \varphi_{j}(z)=0 \\
\left(\frac{\partial^{2}}{\partial x^{2}}+\frac{\partial^{2}}{\partial y^{2}}+k^{2}\right) \chi_{j}=0
\end{gathered}
$$

with

$$
k^{2}=k_{x}^{2}+k_{y}^{2}
$$

The Bernoulli equation together with the solutions to Eqs. (11) are the basic ingredients for calculating the ripplon modes in the following two subsections.

\section{A- One hard-wall case}

Assume that the hard-wall is located at $\mathrm{z}=-\mathrm{h}$ as depicted in Fig. 1.

The geometrical configuration of the system suggests that the reasonable conditions to be imposed on the two condensates are

which lead to

$$
\begin{gathered}
\left.\frac{\partial \varphi_{2}(z)}{\partial z}\right|_{z=-h}=0, \\
\varphi_{1}(z) \rightarrow 0 \text { as } z \rightarrow+\infty,
\end{gathered}
$$

$$
\delta \phi_{1}=\left(A_{1} \cos \sigma+B_{1} \sin \sigma\right) \exp (-k z)
$$




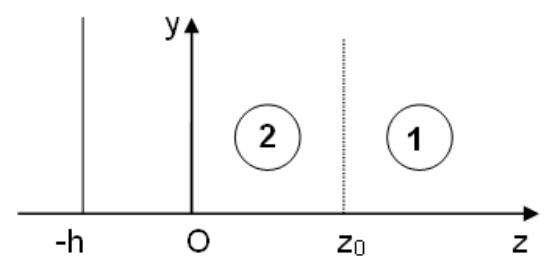

Fig. 1. The interface is located at $z=z_{0}$ and the hard wall at $z=-h$.

$$
\delta \phi_{2}=\left(A_{2} \cos \sigma+B_{2} \sin \sigma\right) \cosh [k(z+h)],
$$

where $A_{j}, B_{j}$ are small parameters.

For simplicity let us restrict to the case

$$
\begin{gathered}
\delta \phi_{1}=A_{1} \exp (-k z) \cos \sigma, \\
\delta \phi_{2}=A_{2} \cosh [k(z+h)] \cos \sigma .
\end{gathered}
$$

Note that the results to be obtained later remain unchanged if $\cos \sigma$ in (12) is replaced by $\sin \sigma$. The kinetic condition at the interface reads

$$
\frac{\partial \eta(\sigma)}{\partial t}=\frac{\hbar}{m_{1}}\left(\frac{\partial \delta \phi_{1}}{\partial z}\right)_{z=z_{0}}=\frac{\hbar}{m_{2}}\left(\frac{\partial \delta \phi_{2}}{\partial z}\right)_{z=z_{0}} .
$$

Inserting (12) into (13) yields

$$
-\omega \frac{d \eta(\sigma)}{d \sigma}=-A_{1} \frac{\hbar k}{m_{1}} \exp \left(-k z_{0}\right) \cos \sigma=A_{2} \frac{\hbar k}{m_{2}} \sinh \left[k\left(z_{0}+h\right)\right] \cos \sigma .
$$

The solution to Eqs. (14) provides immediately

$$
\eta(\sigma)=\eta_{0} \sin \sigma
$$

and

$$
\begin{gathered}
A_{1}=\frac{\eta_{0} m_{1} \omega \exp \left(k z_{0}\right)}{\hbar k}, \\
A_{2}=-\frac{\eta_{0} m_{2} \omega}{\hbar k \sinh \left[k\left(z_{0}+h\right)\right]} .
\end{gathered}
$$

Eqs. (14) are justified for $\left|k \eta_{0}\right| \ll 1$.

Substituting (10), (12), (15), (16) into the Bernoulli equation (5) we arrive at

$$
\omega^{2}=\frac{\alpha k^{3}}{\rho_{2} \operatorname{coth}\left[k\left(z_{0}+h\right)\right]+\rho_{1}},
$$

here $\rho_{1}=m_{1} n_{10}\left(z_{0}+0\right), \rho_{2}=m_{2} n_{20}\left(z_{0}-0\right)$.

As $h$ tends to infinity Eq. (17) turns out to be

$$
\omega=\sqrt{\frac{\alpha}{\rho_{1}+\rho_{2}}} k^{3 / 2},
$$


which is the well known formula for dispersion relation of ripplon in classic hydrodynamics [26, 27]. At the low momentum limit Eq. (17) behaves like

$$
\omega^{2} \approx \frac{\alpha\left(h+z_{0}\right)}{\rho_{2}} k^{4}
$$

which is the main result of this subsection.

In order to get a deeper insight into the issue let us extend to the case when condensates flow with velocity $\vec{V}_{j}$ parallel to the interface. Then the corresponding stationary state is also represented in the form (7b) with

$$
\phi_{j}=-\frac{g_{j j} n_{j 0}}{\hbar} t+\frac{m_{j}}{\hbar} \vec{V}_{j} \cdot \vec{r}_{\perp}+\delta \phi_{j}
$$

where $\delta \phi_{j}$ is given by Eqs. (12). In this case the kinetic condition at the interface (13) is modified as

$$
\left(\frac{\partial}{\partial t}+\overrightarrow{V_{j}} \frac{\partial}{\partial \overrightarrow{r_{\perp}}}\right) \eta=\frac{\hbar}{m_{j}}\left(\frac{\partial \delta \phi_{j}}{\partial z}\right)_{z=z_{0}}
$$

Substituting (12) and (15) into (19) gives

$$
\begin{gathered}
A_{1}=\frac{\eta_{0} m_{1}\left(\omega-\vec{V}_{1} \vec{k}\right) \exp \left(k z_{0}\right)}{\hbar k}, \\
A_{2}=-\frac{\eta_{0} m_{2}\left(\omega-\vec{V}_{2} \vec{k}\right)}{\hbar k \sinh \left[k\left(z_{0}+h\right)\right]} .
\end{gathered}
$$

After inserting (20) into (5) we are led to the equation

$$
\rho_{1}\left(\omega-\vec{V}_{1} \vec{k}\right)^{2}+\rho_{2} \operatorname{coth}\left[k\left(z_{0}+h\right)\right]\left(\omega-\vec{V}_{2} \vec{k}\right)^{2}=\alpha k^{2},
$$

yielding

$$
\omega_{ \pm}=V_{c} k \pm \frac{k}{\sqrt{\rho_{1}+\rho_{2}}} \sqrt{\alpha k-\frac{\rho_{1} \rho_{2}}{\rho_{1}+\rho_{2}} V_{r}^{2}}
$$

here

$$
V_{c}=\frac{\rho_{1} V_{1} \cos \theta_{1}+\rho_{2} \operatorname{coth}\left[k\left(z_{0}+h\right)\right] V_{2} \cos \theta_{2}}{\rho_{1}+\rho_{2} \operatorname{coth}\left[k\left(z_{0}+h\right)\right]},
$$

with $\theta_{j}=\widehat{\left(\vec{V}_{j}, \vec{k}\right)}$.

It is easily seen that as $h$ tends to infinity (21) turns out to be

$$
\omega_{ \pm}=V_{c} k \pm \sqrt{\frac{\alpha k^{3}}{\rho_{1}+\rho_{2}}-\frac{\rho_{1} \rho_{2} V_{r}^{2} k^{2}}{\left(\rho_{1}+\rho_{2}\right)^{2}}}
$$

which is a well known result.

The small-k behavior of (21) reads

$$
\omega \approx V_{2} \cos \theta_{2} k \pm \sqrt{-\frac{\rho_{1}}{\rho_{2}}\left(h+z_{0}\right)} V_{r} k^{3 / 2} .
$$

Eq. (23) indicates that the Kelvin-Helmholtz instability always occurs for $V_{r}=\left|\vec{V}_{1}-\vec{V}_{2}\right|>0$. Next let us go over to the two hard-wall case. 


\section{B- Two hard-wall case}

Assume that the condensate 1 (condensate 2) resides in the region $z>z_{0}\left(z<z_{0}\right)$ and the hard wall 1 (2) is located at $z=h_{1}\left(z=-h_{2}\right)$ as plotted in Fig. 2. Then the boundary conditions we impose at the two hard walls are the Dirichlet ones

$$
\begin{gathered}
\left.\frac{\partial \varphi_{1}(z)}{\partial z}\right|_{z=h_{1}}=0 \\
\left.\frac{\partial \varphi_{2}(z)}{\partial z}\right|_{z=-h_{2}}=0 .
\end{gathered}
$$

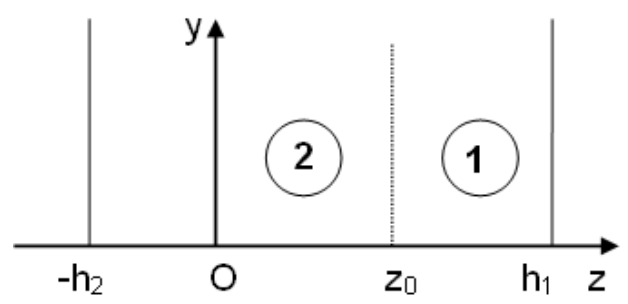

Fig. 2. The interface is located at $z=z_{0}$ and two hard walls at $z=h_{1}$ and $z=-h_{2}$, respectively.

The solutions of Eqs. (11) fulfilling the foregoing conditions read

$$
\begin{aligned}
& \delta \phi_{1}=\left(C_{1} \cos \sigma+D_{1} \sin \sigma\right) \cosh \left[k\left(h_{1}-z\right)\right], \\
& \delta \phi_{2}=\left(C_{2} \cos \sigma+D_{2} \sin \sigma\right) \cosh \left[k\left(z+h_{2}\right)\right],
\end{aligned}
$$

in which $C_{j}, D_{j}$ are small parameters.

Without loss of generality we can use only the solutions

$$
\begin{aligned}
\delta \phi_{1} & =C_{1} \cosh \left[k\left(h_{1}-z\right)\right] \cos \sigma, \\
\delta \phi_{2} & =C_{2} \cosh \left[k\left(z+h_{2}\right)\right] \cos \sigma,
\end{aligned}
$$

in what follows. Substituting (24) into the kinetic condition at the interface (13) we obtain

$$
\begin{gathered}
C_{1}=\frac{\eta_{0} m_{1} \omega}{\hbar k \sinh \left[k\left(h_{1}-z_{0}\right)\right]}, \\
C_{2}=-\frac{\eta_{0} m_{2} \omega}{\hbar k \sinh \left[k\left(z_{0}+h_{2}\right)\right]},
\end{gathered}
$$

which together with the Bernoulli equation (5) give

$$
\omega^{2}=\frac{\alpha k^{3}}{\rho_{1} \operatorname{coth}\left[k\left(h_{1}-z_{0}\right)\right]+\rho_{2} \operatorname{coth}\left[k\left(z_{0}+h_{2}\right)\right]},
$$

taking the familiar form

$$
\omega=\sqrt{\frac{\alpha}{\rho_{1}+\rho_{2}}} k^{3 / 2}
$$

as $h_{1}, h_{2}$ tend to infinity. 
At the low momentum limit (25) behaves like

$$
\omega^{2} \approx \frac{\alpha\left(z_{0}+h_{2}\right)\left(h_{1}-z_{0}\right)}{\rho_{1}\left(z_{0}+h_{2}\right)+\rho_{2}\left(h_{1}-z_{0}\right)} k^{4} .
$$

Eq. (26) is the main result of this subsection.

Finally, let us extend to the case when the j-component flows with velocity $\vec{V}_{j}$ parallel to the interface. The calculation analogous to what we did before provides

$$
\omega_{ \pm}=V_{c} k \pm \sqrt{\frac{\alpha k^{3}}{\rho_{1} \operatorname{coth}\left[k\left(h_{1}-z_{0}\right)\right]+\rho_{2} \operatorname{coth}\left[k\left(z_{0}+h_{2}\right)\right]}-\frac{\rho_{1} \rho_{2} \operatorname{coth}\left[k\left(h_{1}-z_{0}\right)\right] \operatorname{coth}\left[k\left(z_{0}+h_{2}\right)\right] V_{r}^{2} k^{2}}{\left(\rho_{1} \operatorname{coth}\left[k\left(h_{1}-z_{0}\right)\right]+\rho_{2} \operatorname{coth}\left[k\left(z_{0}+h_{2}\right)\right]\right)^{2}}},
$$

here

$$
V_{c}=\frac{\rho_{1} V_{1} \cos \theta_{1} \operatorname{coth}\left[k\left(h_{1}-z_{0}\right)\right]+\rho_{2} V_{2} \cos \theta_{2} \operatorname{coth}\left[k\left(z_{0}+h_{2}\right)\right]}{\rho_{1} \operatorname{coth}\left[k\left(h_{1}-z_{0}\right)\right]+\rho_{2} \operatorname{coth}\left[k\left(z_{0}+h\right)\right]} .
$$

It is easy to check that Eq. (27) will get the form (22) when letting $h_{1}, h_{2}$ tend to infinity. The small-k behavior of (27) reads

$$
\omega \approx\left(\frac{\left(h_{1}-z_{0}\right) \rho_{2} V_{2} \cos \theta_{2}+\left(z_{0}+h_{2}\right) \rho_{1} V_{1} \cos \theta_{1}}{\left(h_{1}-z_{0}\right) \rho_{2}+\left(z_{0}+h_{2}\right) \rho_{1}} \pm \frac{V_{r} \sqrt{-\rho_{1} \rho_{2}\left(h_{1}-z_{0}\right)\left(z_{0}+h_{2}\right)}}{\left(h_{1}-z_{0}\right) \rho_{2}+\left(z_{0}+h_{2}\right) \rho_{1}}\right) k .
$$

Eq. (28) indicates that the Kelvin-Helmholtz instability always occurs for $V_{r}=\left|\vec{V}_{1}-\vec{V}_{2}\right|>0$.

\section{CONCLUSION}

In the foregoing section we obtained the main results as follows:

- Due to the spatial restriction the dispersion relation of ripplon is changed, it is of the form $\omega^{2} \approx k^{4}$, instead of $\omega \approx k^{3 / 2}$.

- When the two condensates flow in parallel to the interface the ripplon dispersion relation turns out to be $\omega \approx k$ and the system becomes unstable.

We derived the above results starting from the assumption that the condensates are incompressible. However, it is important to note that taking into account the compressibility means that in the expressions found previously we have to add the correction of the orders of Mach number $\left(\frac{v}{v_{s}}\right)^{2}$ [29], here $v$ is the typical speed of the system and $v_{s}$ the speed of sound in fluid. In the low momentum limit this ratio can be treated as negligibly small, of course. Therefore, the full picture of the system remains unchanged at low energy with the involvement of compressibility . Hence, our results are entirely reliable for guiding the numerical computation of ripplon dispersion relation in a system of two segregated BECs.

Last but not least, it is interesting to note that for systems of two segregated BECs limited by two hard walls the ripplon dispersion relation similar to (25) was derived in Refs. [19,30] by means of the perturbative approximation to GP equations. 


\section{ACKNOWLEDGMENT}

This work is funded by the Vietnam National Foundation for Science and Technology Development (NAFOSTED) under Grant No. FWO.103.2013.09 within the framework of FWONAFOSTED cooperation. This article is also funded from science and technology fund of Pedagogical University Hanoi 2, code C.2017.01. The discussion with Pham The Song is thankfully acknowledged.

\section{REFERENCES}

[1] C. J. Myatt, E. A. Burt, R. W. Ghrist, E. A. Cornell, and C. E. Wieman, Phys. Rev. Lett. 78, 586 (1997).

[2] D. S. Hall, M. R. Matthews, J. R. Ensher, C. E. Wieman and E.A.Cornell, Phys. Rev. Lett. 81 , 1539 (1998).

[3] D. M. Stamper-Kurn, H. J. Miesner, A. P. Chikkatur, S. Inouye, J. Stenger and W. Ketterle, Phys. Rev. Lett. 83, 61 (1999).

[4] M. R. Matthews, B. P. Anderson, P. C. Haljan, D. S. Hall, C. E. Wieman and E. A. Cornell, Phys. Rev. Lett. 83, 2498 (1999).

[5] H. J. Miesner, D. M. Stamper-Kurn, J. Stenger, S. Inouye, A. P. Chikkatur and W. Ketterle, Phys. Rev. Lett. 82, 2228 (1999).

[6] D. J. McCarron, H. W. Cho, D. L. Jenkin, M. P. Koppinger and S. L. Cornish, Phys. Rev. A 84, 011603(R) (2011).

[7] S. B. Papp, J. M. Pino and C. E. Wieman, Phys. Rev. Lett. 101, 040402 (2008).

[8] D. S. Hall, in Emergent Nonlinear Phenomena in Bose-Einstein condensates, edited by P.G.Kevrekidis, D. J. Frantzeskakis and R. Carretero-Gonzales ( Springer-Verlag, Berlin, (2008 ), Chap.16, p.307.

[9] E. Timmermans, Phys. Rev. Lett. 81, 5718 (1998).

[10] P.Ao and S.T.Chui, Phys. Rev. A 58, 4836, (1998).

[11] A. Bezett, V. Bychkov, E. Lundh, D. Kobyakov, and M.Marklund, Phys. Rev. A 82, 043623 (2010).

[12] D. Kobyakov, V. Bychkov, E. Lundh, A. Bezett, V. Akkerman and M. Marklund, Phys. Rev. A 83, 043623 (2011).

[13] J. O. Indekeu and B. Van Schaeybroeck, Phys. Rev. Lett . 93, 210402 (2004).

[14] B. Van Schaeybroeck, Phys. Rev. A 78023624 (2008); 80, 06560(addendum ) (2009); 93, 210402 (2004).

[15] B. Van Schaeybroeck and J. O. Indekeu, Phys. Rev. A 91, 013626 (2015).

[16] J. O. Indekeu, Chang-You Lin, N. V. Thu, B. Van Schaeybroeck and T. H. Phat, Phys. Rev. A 91, 033615 (2015).

[17] K. Sasaki, N. Suzuki and H. Saito, Phys. Rev. A 83, 033602 (2011); 83, 053606 (2011).

[18] C. Ticknor, Phys. Rev. A 89, 053601 (2014).

[19] D. A. Takahashi, M. Kobayashi and M. Nitta, Phys. Rev. B 91, 184501 (2015).

[20] T. Kadokura, T. Aioi, K. Sasaki, T. Kishimoto and H. Saito, Phys. Rev. A 85, 013602 (2012).

[21] F. V. Pepe, P. Facchi, G. Florio and S. Pascazio, Phys. Rev. A 86, 023629 (2012).

[22] T. H. Phat, L. V. Hoa, N. T. Anh and N. V. Long, Ann. Phys.(NY). 324, 2074 (2009) .

[23] B. Van Schaeybroeck, Phys. Rev. A 392, 3806 (2013).

[24] A. Roy, S. Gautam and D. Angom, Phys. Rev. A 89, 013617 (2014).

[25] G. Ceccarelli, J. Nespolo, A. Pelissetto and E. Vicari, Phys. Rev. A 92, 043613 (2015).

[26] L. D. Landau and E. M. Lifshitz, Fluid Mechanics, 4th ed. (Academic Press, NY, 2008).

[27] I. K. Kundu, I. M. Cohen and D. R. Dowling, Fluid Mechanics, 5th edition, Elsevier 2012, Amsterdam, Netherlands.

[28] This approximation corresponds to the Boussinesq approximation in classic hydrodynamics, see [27], p.135 for detail .

[29] A. N. Malmi - Kakkada, O. T. Valls and C. Dasgupta, J. Phys. B 47, 055301 (2014).

[30] D. A. Takahashi and M. Nitta, Ann. Phys.(NY). 354, 101 (2015). 